\title{
EDITORIAL
}

\section{Antimicrobial Resistance: Are We Heading Towards Post Antibiotic Era?}

The discovery of penicillin in 1928 by Alexander Fleming, a magic antibiotic used for treatment of most of the bacterial infection marked the beginning of antibiotic era. With booming drug development a new drug was always available to treat increasing drug resistant bacteria. Between 1935 to 2003 fourteen classes of antibiotics were developed. However, with the indiscriminate and inappropriate use of antibiotics, microbes developed mechanisms to elude the action of antimicrobial agents. Microbes are cleverer than man and they develop strategies rapidly to overcome action of new antimicrobial agent. It seems that drug versus bug battle will never end. However if we want to win this war we have to be a step ahead and develop new drug molecules at a much faster pace. Unfortunately this is not possible as it takes more than a decade from discovery of new molecule, its purification, clinical trials and approval before it can be used. Since 1998 only 10 new antibiotics have been approved, only 2 of which (Linezolid and Daptomycin) are actually in clinical use. Antibiotics currently in development are broader spectrum in nature, which means they are likely to further promote development of resistance if approved and used. The alarming increase in MDR pathogens has led the infectious disease society of America's Bad Bugs, No drug task force to call for global commitment from stakeholders to support the development of 10 new drugs by 2020.This so- called 10x20 initiatives has been likened to John F. Kennedy's dream of walking on moon. Until this next giant step is achieved, those of us not developing new drugs have another job: conserve antibiotics we have. Unfortunately, current practice and likely future trends in hospital and community antibiotic prescribing give much cause for concern.

The resistance to antibiotics is increasing at a faster pace than it can be controlled. ${ }^{1}$ It is one of the major public health problems especially in developing countries where easy availability and higher consumption of medicines have lead to disproportionately higher incidence of inappropriate use of antibiotics and greater levels of resistance. ${ }^{2}$ This results in difficulty in controlling the diseases in the community and ineffective delivery of the health care services. ${ }^{3}$ The steady emergence of populations of antimicrobial-resistant pathogenic bacteria resulting from use, misuse, and abuse of antimicrobials has today become a major global health concern. $^{4}$

Antimicrobial resistance genes have been suggested to originate from environmental bacteria, as clinically relevant resistance genes have been detected on the chromosome of environmental bacteria. Urgent measures are required not only to minimize the use of antimicrobials for prophylactic and therapeutic purposes but also to look for alternative strategies for the control of bacterial infections ${ }^{4}$

The resistance spectrum of pathogens varies in different regions. Therefore local resistance patterns have to be known for appropriate antimicrobial use. ${ }^{5}$ Various studies in India have highlighted the drug resistance pattern like multidrug resistant Extended-Spectrum $\beta$-Lactamase producing Klebsiella pneumoniae, Ciprofloxacin resistant Salmonella Typhi, emergence of vancomycin-intermediate staphylococci, fluoroquinolone resistance among Salmonella Paratyphi A, Pseudomonas aeruginosa and Acinetobacter baumannii resistant to ceftazidime, cefepime and ciprofloxacin. ${ }^{3}$

Extended-spectrum $\beta$-lactamases (ESBL) are the ones most often encountered in the hospital (intensive care) setting. Methicillin-resistant Staphylococcus aureus (MRSA) and vancomycin-resistant enterococci (VRE) have also been found to have a significant nosocomial ecology. ${ }^{6}$ The advent of community-acquired MRSA will also put tremendous pressure on primary care and emergency department doctors to broaden their choice of antibiotic prescription for skin soft tissue infections. Carbapenems are the last line of defense against the non-Enterobacteriaceae pathogens, such as Pseudomonas aeruginosa and Acinetobacter baumannii .However, since the first description of the blaOXA genes, there has been a worldwide increase in the dissemination of new resistance determinants conferring carbapenem resistance. ${ }^{7}$ Also Community outbreaks of 
ESBL producers will lead to increased carbapenem use. This issue is perhaps the most problematic for hospital practice given the absence of likely alternatives to carbapenems for treatment of serious hospital acquired infections by carbapenemase producers. In many hospitals, quinolone resistance in Enterobacteriaceae and Pseudomonas aeruginosa is now at such a high level that quinolones cannot be relied on for empirical monotherapy. For similar reasons neither can broad-spectrum cephalosporins. Even carbapenems are under serious threat in many countries and carbapenemase-producing, polymyxin- and aminoglycoside resistant $P$. aeruginosa and Acinetobacter baumannii are increasingly described. In our hospital $>40 \%$ isolates of Staphylococcus aureus are MRSA and majority of gram negative particularly Pseudomonas aeruginosa, Klebsiella pneumoniae and Acinetobacter are multidrug resistant. This is a real doomsday scenario as there are few, if any, drugs currently in development to address this problem. The increased prevalence of MDR, XDR and Pan drug resistance has led to speculate that we are nearing the end of antibiotic era.

\section{Bashir Ahmad Fomda}

Professor Department of Microbiology,

SKIMS, Srinagar

\section{REFERENCES:}

1. Renuka K, Kapil A, Kabra SK, et al. Reduced susceptibility to ciprofloxacin and gyra gene mutation in north Indian strains of Salmonella enterica serotype Typhi and serotype Paratyphi A. Microb Drug Resist. 2004; 10: 146-53.

2. The world health report. World Health Organization, Geneva: 1996.

3. Kumar SG, Adithan C, Harish BN, Sujatha S, Roy G, Malini A.Antimicrobial resistance in India: A review. J Nat Sci Biol Med. 2013 Jul;4(2):286-91.

4. L. Cantas, Syed Q. A. Shah, L. M. Cavaco, C. M. Manaia, F. Walsh, M. Popowska, H. Garelick et al. A brief multi-disciplinary review on antimicrobial resistance in medicine and its linkage to the global environmental microbiota. Front. Microbiol., 2013:4:96.

5. Raghunath D. Emerging antibiotic resistance in bacteria with special reference to India. J Biosci. 2008;33:593-603.

6. Otter J. A, French G. L. Molecular epidemiology of community-associated methicillin resistant Staphylococcus aureus in Europe. Lancet (KPC) Infect. Dis. 2010 ; 10: 227-239.

7. Brown S, Bantar C, Young H. K, Amyes S. G. B. Limitation of Acinetobacter baumannii treatment by plasmid-mediated carbapenemase ARI-2. Lancet 1998; 351: 186-187. 